\title{
Improving Fatigue Resistance of Dihydropyrene by Encapsulation within a Coordination Cage
}

\author{
Martina Canton, ${ }^{\nabla}$ Angela B. Grommet, ${ }^{\nabla}$ Luca Pesce, Julius Gemen, Shiming Li, Yael Diskin-Posner, \\ Alberto Credi, Giovanni M. Pavan, Joakim Andréasson, and Rafal Klajn*
}

Cite This: J. Am. Chem. Soc. 2020, 142, 14557-14565

Read Online

\section{ACCESS | Lill Metrics \& More | 回 Article Recommendations ｜（） Supporting Information}

ABSTRACT: Photochromic molecules undergo reversible isomerization upon irradiation with light at different wavelengths, a process that can alter their physical and chemical properties. For instance, dihydropyrene (DHP) is a deep-colored compound that isomerizes to light-brown cyclophanediene (CPD) upon irradiation with visible light. CPD can then isomerize back to DHP upon irradiation with UV light or thermally in the dark. Conversion between DHP and CPD is thought to proceed via a biradical intermediate; bimolecular events involving this unstable intermediate thus result in rapid decomposition and poor cycling performance. Here, we show that the reversible isomerization of DHP can be stabilized upon confinement within a $\mathrm{Pd}_{6}^{\mathrm{II}} \mathrm{L}_{4}$

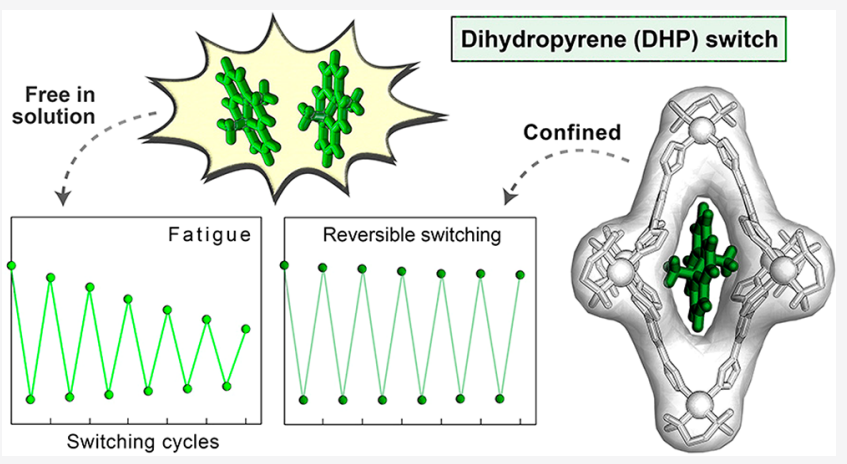
coordination cage. By protecting this reactive intermediate using the cage, each isomerization reaction proceeds to higher yield, which significantly decreases the fatigue experienced by the system upon repeated photocycling. Although molecular confinement is known to help stabilize reactive species, this effect is not typically employed to protect reactive intermediates and thus improve reaction yields. We envisage that performing reactions under confinement will not only improve the cyclic performance of photochromic molecules, but may also increase the amount of product obtainable from traditionally low-yielding organic reactions.

\section{INTRODUCTION}

Dihydropyrene (DHP) belongs to the family of diarylethene photoswitches; ${ }^{1,2}$ this switch consists of a large $\pi$-conjugated system $^{3}$ that can be converted to the open form cyclophanediene (CPD) using visible light. This isomerization is reversible, as CPD can be converted back to DHP both photochemically and thermally. ${ }^{4}$ Unlike other photochromic systems, such as azobenzenes and spiropyrans, DHP is a rare example of a compound where the colored form constitutes the thermodynamically more stable isomer. ${ }^{5}$ One disadvantage of this molecular switch, however, is its instability and poor fatigue resistance. The observed fatigue is thought to arise from the reactivity of the biradical intermediate formed as a result of photocleavage of the central $\mathrm{C}-\mathrm{C}$ bond in the singlet excited state of DHP. ${ }^{6,7}$ To render photoswitching cycles more robust, we propose stabilizing the reaction under confinement, ${ }^{8,9}$ by encapsulating DHP within the cavity of a coordination cage. $^{10-14}$

Reactive species such as radicals ${ }^{15-18}$ and photochromes $^{19-25}$ can be stabilized under confinement through a range of different mechanisms. ${ }^{26-29}$ Confined spaces can, for instance, create a hermetic seal around a reactive species, which prevents or minimizes interactions with the external environment. $^{30-32}$ By confining DHP within a coordination cage, we hoped to protect the unstable intermediate from oxidation and radical-radical interactions that lead to decomposition. Octahedral $\mathrm{Pd}_{6}^{\mathrm{II}} \mathrm{L}_{4}$ cage 1 has been previously shown to be highly flexible due to the rotational freedom around the imidazole-benzene bonds. ${ }^{33}$ Cage 1 can thus adapt the shape of its cavity to the geometry of bound guest molecules. Furthermore, the flexibility of cage $\mathbf{1}$ allows the cavity to dynamically adapt in response to the isomerization of encapsulated spiropyran ${ }^{33}$ and azobenzene ${ }^{34}$ photoswitches.

Here, we show that cage $\mathbf{1}$ encapsulates DHP in quantitative yield, forming a 1:1 inclusion complex. Upon irradiation with blue light, encapsulated DHP isomerizes to CPD within the cage and maintains a similar orientation within the cage cavity before and after the reaction. Furthermore, the back-reaction from CPD to DHP was achieved within the cage both upon irradiation with UV light and in the dark. Using atomistic molecular dynamics simulations, we show that encapsulated

Received: June 7, 2020

Published: August 14, 2020 


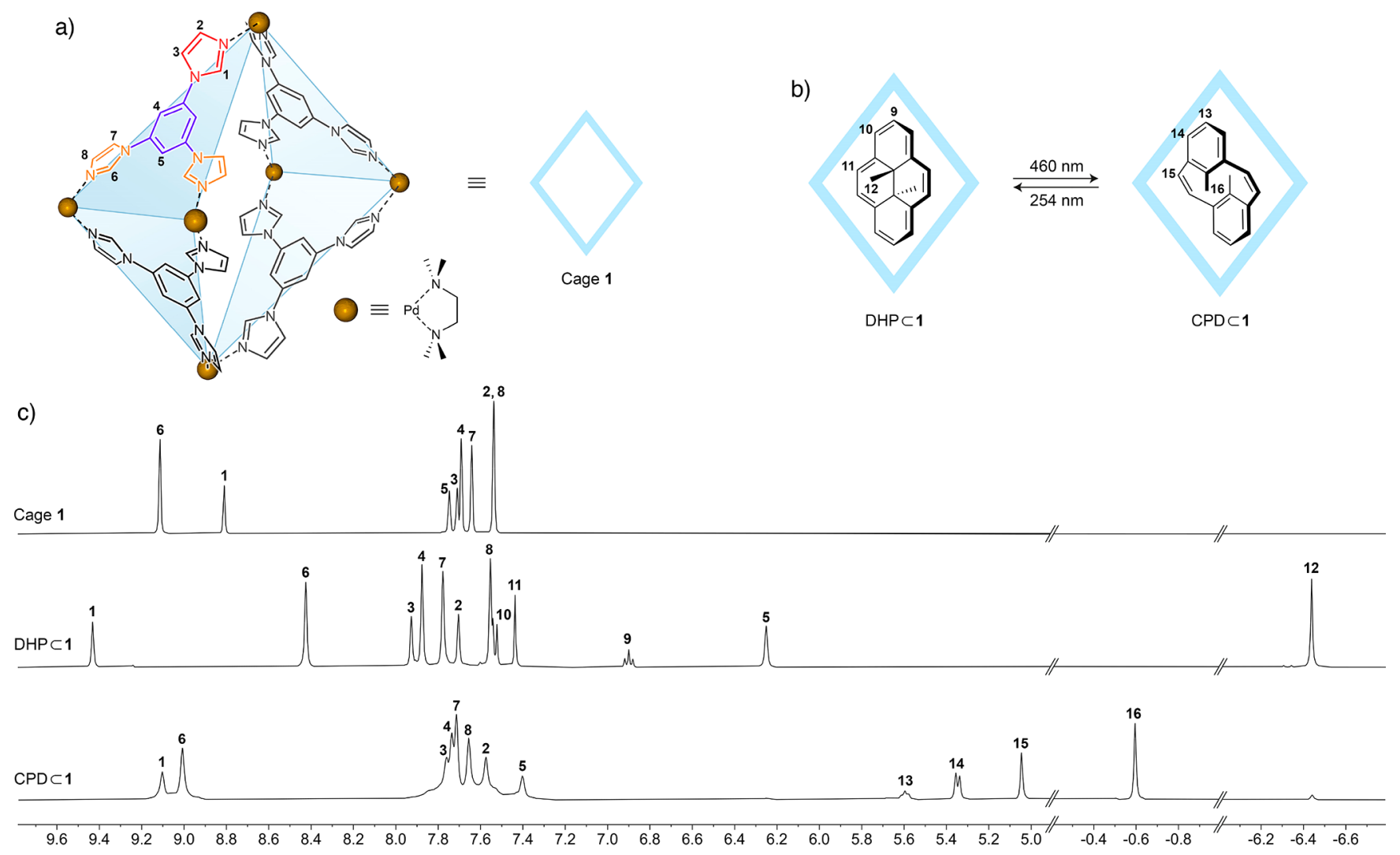

Figure 1. (a) Structural formula of cage 1, including atom numbering scheme used in the NMR spectra. (b) Reversible photoisomerization

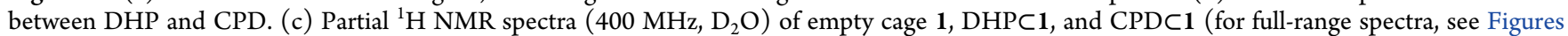
S2, S7, and S17).

DHP behaves like a molecular gyroscope within the cage cavity, whereas the movement of encapsulated CPD is relatively hindered. We found that while confinement within the cage does not greatly influence the rate of switching, it significantly increases the fatigue resistance of DHP switching, as compared to free DHP in solution. To the best of our knowledge, this work constitutes a unique example in which stabilization of a reactive intermediate by a coordination cage is employed as a technique to improve the yield of a reversible reaction.

\section{RESULTS AND DISCUSSION}

To study the behavior of DHP under confinement, DHP was first encapsulated within cage 1. To prepare this complex, excess solid DHP was added to an aqueous solution of 1 (1 $\mathrm{mM}$ ) and stirred for $24 \mathrm{~h}$ at room temperature. Undissolved DHP was then removed using ultracentrifugation. Despite DHP being insoluble in water, the resulting supernatant was observed to be green, which indicated the presence of encapsulated DHP.

The characterization of this complex was achieved by performing $1 \mathrm{D}$ and a suite of $2 \mathrm{D}$ NMR experiments. The ${ }^{1} \mathrm{H}$ DOSY spectrum indicates that all signals observable by ${ }^{1} \mathrm{H}$ NMR diffuse at the same rate (Figure S9); that is, the signals attributed to DHP and to cage 1 diffuse together through solution, which confirms the formation of the complex. All signals observed by ${ }^{1} \mathrm{H}$ NMR were fully assigned by ${ }^{1} \mathrm{H}-{ }^{1} \mathrm{H}$ COSY and ${ }^{1} \mathrm{H}-{ }^{1} \mathrm{H}$ NOESY (Figures $\mathrm{S} 10$ and S11). The ${ }^{1} \mathrm{H}$ NMR spectrum of DHPC1 (Figure 1) shows that the aromatic peaks from DHP $\left(\mathrm{H}_{9}, \mathrm{H}_{10}, \mathrm{H}_{11}\right)$ are shifted upfield with respect to those from free DHP in chloroform (Figures S6 and S8). Furthermore, the methyl peak $\left(\mathrm{H}_{12}\right)$ from encapsulated DHP is shifted significantly upfield from -4.24 to $-6.44 \mathrm{ppm}$ upon encapsulation; these effects are due to shielding by the aromatic panels of the cage. Moreover, integrating signals from the cage and from encapsulated DHP indicates that the binding stoichiometry is $1: 1$.

To confirm the binding stoichiometry of this inclusion complex, an aqueous solution of the cage in water $(0.62 \mathrm{mM})$ was titrated using a solution of DHP dissolved in acetonitrile (6.94 mM) (Supporting Information, section 6). The formation of the complex was followed by UV/vis absorption spectroscopy. The absorption signals attributed to encapsulated DHP increased in absorbance until 1 equiv of DHP was added. Upon further addition of DHP, a plateau in the absorbance of DHPC1 was achieved (Figure S12b). Furthermore, the baseline was observed to increase, which indicated that excess DHP was not incorporated within the cage cavity and thus began to precipitate from solution (Figure $\mathrm{S} 12 \mathrm{a}, \mathrm{c})$. As compared to the UV/vis spectra of free DHP in acetonitrile $\left(\lambda_{\max }=336\right.$ and $\left.376 \mathrm{~nm}\right)$, pentane $\left(\lambda_{\max }=336\right.$ and $375 \mathrm{~nm})$, and toluene $\left(\lambda_{\max }=340\right.$ and $\left.379 \mathrm{~nm}\right)$, the wavelengths of maximum absorption from encapsulated DHP in water $\left(\lambda_{\max }=346\right.$ and $\left.383 \mathrm{~nm}\right)$ are red-shifted by 10 and 7 $\mathrm{nm}, 10$ and $8 \mathrm{~nm}$, and 6 and $4 \mathrm{~nm}$, respectively; this effect is likely due to differences in the intermolecular interactions between free DHP and the solvent, and between encapsulated DHP and the aromatic panels of the cage. We note that there is no solvent that can dissolve both DHPC1 and free DHP, 
which renders direct comparison between these two systems challenging.

${ }^{1} \mathrm{H}-{ }^{1} \mathrm{H}$ NOESY provided additional information elucidating the orientation of DHP within the cavity of cage 1 . The methyl groups $\left(\mathrm{H}_{12}\right)$ from DHP are correlated with the equatorial and benzene regions of the cage $\left(\mathrm{H}_{4-8}\right)$, highlighted in purple and orange in Figure 2a. This result suggests that DHP is oriented
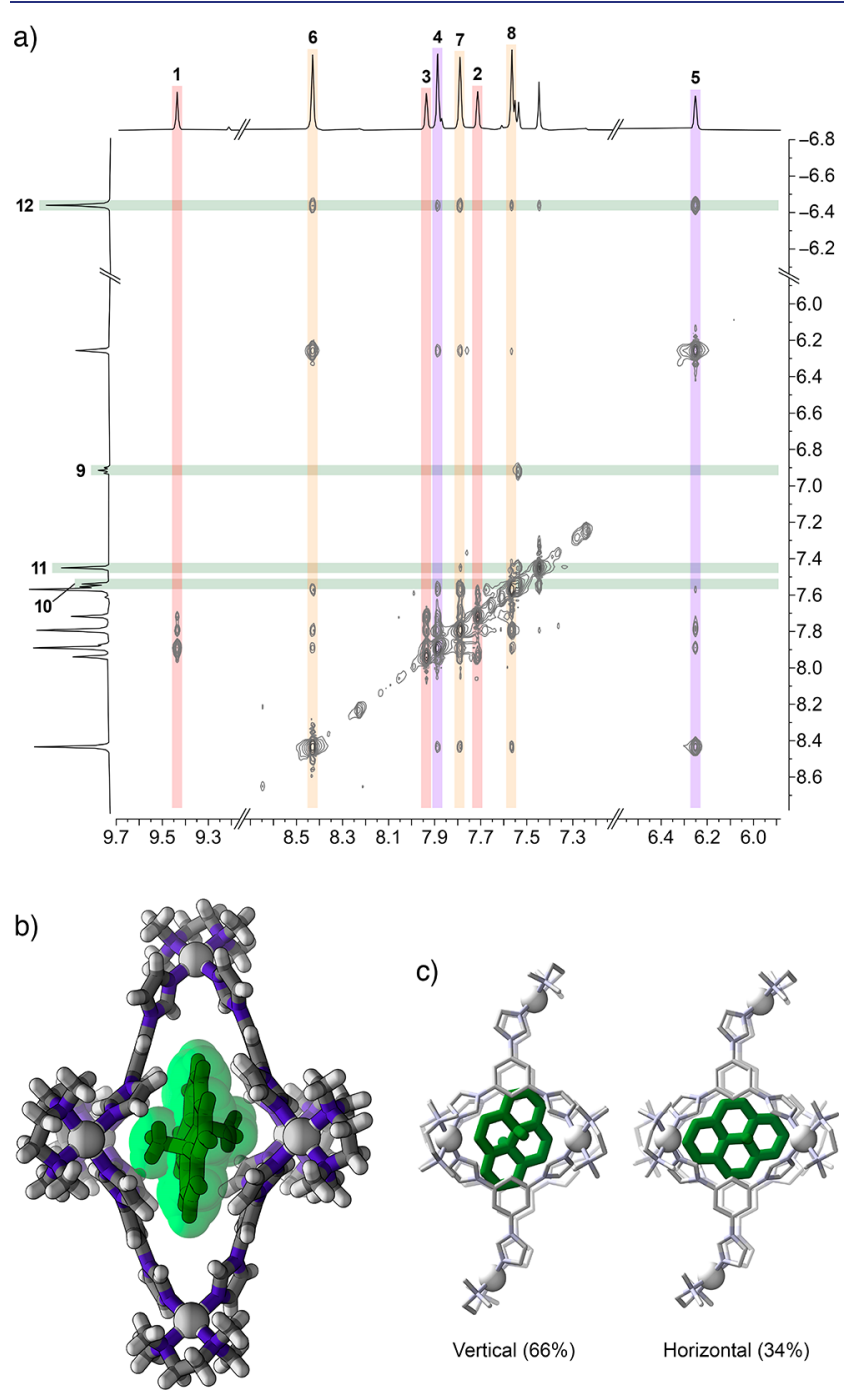

Figure 2. (a) Partial ${ }^{1} \mathrm{H}-{ }^{1} \mathrm{H}$ NOESY spectrum $\left(500 \mathrm{MHz}, \mathrm{D}_{2} \mathrm{O}\right)$ of $\mathrm{DHP} \subset 1$. Signals highlighted in red, orange, and purple originate from 1's axial imidazole, equatorial imidazole, and benzene rings, respectively (see Figure 1a). Signals highlighted in green originate from DHP. (b) Crystal structure of DHPC1 (major conformation). (c) Side-views of the crystal structures of the major and minor conformers of DHPC1 showing the orientation of DHP within cage 1.

within the cage cavity such that the methyl groups are accommodated within the widest, equatorial region of the cage. No significant correlations are observed between the aromatic protons $\mathrm{H}_{9}-\mathrm{H}_{11}$ from $\mathrm{DHP}$ and the cage. Similarly, no significant correlations are observed between protons from the axial region of the cage $\left(\mathrm{H}_{1-3}\right)$, highlighted in red, and DHP. The absence of these correlations indicates that DHP may be spinning within the cavity of cage 1 , with the methyl groups remaining relatively fixed in the equatorial pockets of the cage cavity. Given that only one set of signals corresponding to encapsulated DHP is observed in the ${ }^{1} \mathrm{H}$ NMR spectrum, we infer that this rotation is fast on the NMR timescale.

The solution-state structure surmised from NOESY correlations was confirmed in the solid state upon obtaining the X-ray crystal structure of DHPC1 (Figure 2b,c). Crystals were obtained by slow evaporation of water from a solution of $\mathrm{DHP} \subset 1$ at room temperature. As suggested by NOESY, disorder was observed in the orientation of DHP within the cavity. Upon refinement of the crystal structure, two orientations of DHP were observed. The major species, present in approximately $66 \%$ composition, contains DHP oriented roughly vertically within the cavity, while the minor species (34\%) contains DHP oriented horizontally (Figure 2c). Furthermore, the X-ray crystal structure demonstrates the high degree of flexibility within the cage, which allows the cavity to conform to the geometry of DHP. As compared to the crystal structure of the empty cage, the structure of DHPC1 is distorted along the axial direction; the axial $\mathrm{Pd}-\mathrm{Pd}$ distance increases from $16.865 \AA$ in the empty cage ${ }^{34}$ to $19.552 \AA$ and $19.910 \AA$ in $\mathrm{DHPC1}$ (the crystal structure features two alternating complexes with slightly different structural parameters), corresponding to $15.9 \%$ and $18.0 \%$ increases, respectively. Previously reported crystal structures of inclusion complexes formed by cage $\mathbf{1}$ also show a similar effect. The crystal structure for $\mathrm{DHP} \subset 1$, however, is significantly more distorted than that for any of the seven inclusion complexes of 1 obtained to date, ${ }^{33-36}$ where the largest increase in the axial $\mathrm{Pd}-\mathrm{Pd}$ distance corresponded to $13.3 \%$ (Table S1).

Having obtained the crystal structure for DHPC1, we noticed a striking similarity with the structure previously reported for an encapsulated merocyanine (MCC1 in Figure 3; Figures S13 and S14). In both structures, cage 1 extends axially to accommodate the guest, and the methyl groups of DHP and MC are located within the equatorial pockets of the cage. Furthermore, encapsulated MC is oriented in a vertical fashion similar to the major species within the structure of DHPC1.

Given the parallels between these two structures, we were interested in whether one of these guests could displace the other from the cavity of $\mathbf{1}$ (Figure 3a). To this end, we treated DHPC 1 with a mixture of 2 equiv of free DHP and 3 equiv of $\mathrm{MCH}$ (i.e., the nonencapsulated form of $\mathrm{MC}^{33}$ ) such that the total amounts of DHP and $\mathrm{MCH}$ in the system were the same (see Supporting Information, section 9). The reaction could conveniently be followed by UV/vis absorption spectroscopy, due to the fact that the encapsulated MC is the only species in the system absorbing at $\lambda>550 \mathrm{~nm}$. Specifically, we followed the increase of absorption at the wavelength of MC's maximum absorption $(600 \mathrm{~nm})$ and found that the exchange proceeded rapidly, reaching equilibrium within the first $30 \mathrm{~min}$ (Figure $3 \mathrm{~b}$ and the green trace in Figure 3d, top). The equilibration could be followed independently by monitoring the fluorescence intensity at $670 \mathrm{~nm}\left(\lambda_{\text {exc }}=550 \mathrm{~nm}\right)$, where encapsulated MC is the exclusive emitter (Figure S15a and the green trace in Figure $3 \mathrm{~d}$, bottom). In a separate experiment, we prepared $\mathrm{MCC} 1$ and mixed it with 2 equiv of free $\mathrm{MCH}+3$ equiv of DHP. The reaction was somewhat slower, which could be attributed to the very low DHP solubility in water; nevertheless, similar compositions of mixtures in both experiments were reached within the initial $1 \mathrm{~h}$ of exchange (Figure $3 \mathrm{~d}$ ). We have previously demonstrated ${ }^{33}$ that cage 1 binds MC with a high association constant of $K_{\text {assoc }}>10^{7} \mathrm{M}^{-1}$. Unfortunately, we cannot directly measure $K_{\text {assoc }}$ for DHP $\subset 1$ because DHP is insoluble in water. Furthermore, upon adding $<1$ equiv of 
a)

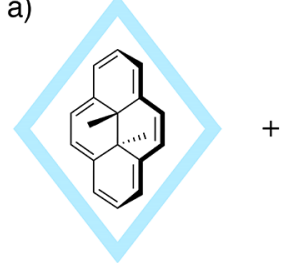

$\mathrm{DHP} \subset 1$
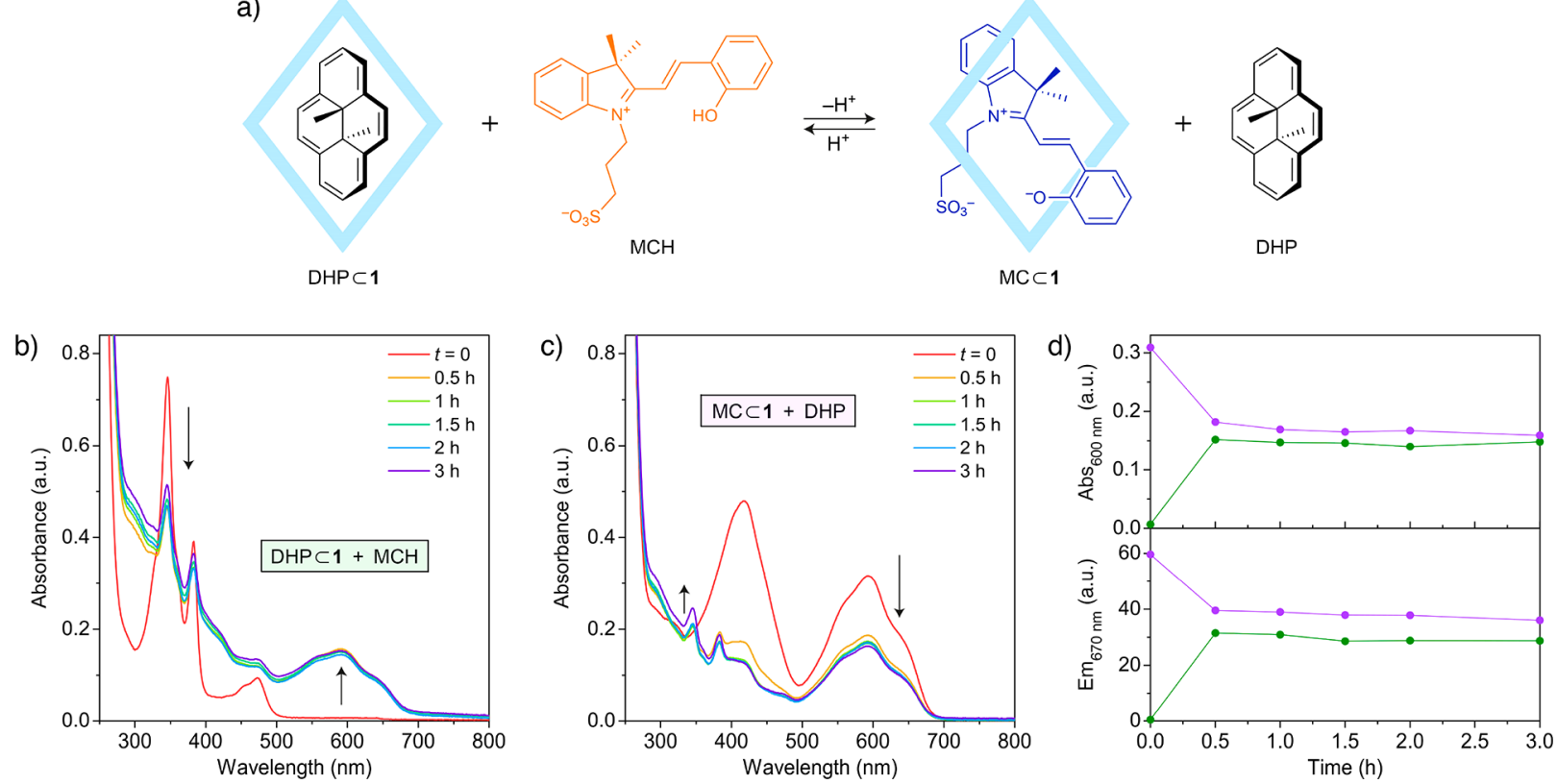

Figure 3. (a) Dynamic guest exchange involving DHP $\subset 1$ and a sulfonated merocyanine. (b) Evolution of UV/vis absorption spectra upon mixing DHPC1 with an equimolar amount of free MCH. (c) Evolution of UV/vis absorption spectra upon mixing MC $\subset 1$ with an equimolar amount of free DHP. (d) Following the absorbance at $600 \mathrm{~nm}$ (top; replotted from parts b and c) and emission at $670 \mathrm{~nm}$ (regions where encapsulated DHP

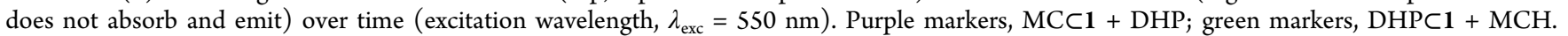

DHP to a solution of cage 1 in water, all DHP becomes encapsulated (Supporting Information, section 6), and the resulting linear titration curve cannot be fit to obtain an accurate $K_{\text {assoc }}$ value. The competition experiment described above, however, can be used to estimate the strength of $K_{\text {assoc }}$ for $\mathrm{DHPC1}$. Provided that equimolar amounts of $\mathrm{MC}$ and DHP were used in our experiments, resulting in a $\sim 1: 1$ distribution of the two guests inside the cage at equilibrium (Figure 3d), we conclude that DHP interacts with the cage with an affinity similar to that of MC. This result arises from several features shared by these guests; both compounds are planar, highly conjugated, and they are similar in geometry, with two methyl groups oriented orthogonally to the aromatic system. As mentioned above, encapsulation of DHP and MC is associated with a similar degree of structural rearrangement by cage 1, which accumulates a similar energetic cost. This cost is offset in part by similar $\pi-\pi$ stacking interactions between the cage panels and the aromatic guests DHP and MC.

Having extensively characterized the DHPC1 complex, we studied DHP photoisomerization by irradiating an aqueous solution of DHPC1 with blue light at $460 \mathrm{~nm}$. The solution of $\mathrm{DHP} \subset 1$ was observed to change from green to light-brown; these visual changes were accompanied by changes in the UV/ vis spectra, which show a consistent decrease in the band centered at $470 \mathrm{~nm}$ in the visible region (Figure $4 \mathrm{a}$ ). We recorded UV/vis spectra every $30 \mathrm{~s}$ and monitored conversion to CPDC1 as a function of irradiation time (Figure $4 \mathrm{~b}$ ). For comparison, we performed the same experiment under identical irradiation conditions on free DHP in acetonitrile (Figure 4c). In both cases, conversion proceeded according to apparent first-order kinetics, with rate constants of $1.5 \times 10^{-2}$ and $0.5 \times 10^{-2} \mathrm{~s}^{-1}$ for free and encapsulated DHP, respectively. From the ratio of these constants, it can be inferred that the DHP $\rightarrow$ CPD photoisomerization quantum yield decreases by a factor of 3 upon encapsulation. At the same time, we note that $\mathrm{CPD}$ requires slightly more space than does DHP, which likely contributes to the decrease in isomerization rate that we observe under confinement. Indeed, these results are consistent with the switching behavior of diarylethenes confined within highly ordered polymer martices. 37

We also followed the reaction by ${ }^{1} \mathrm{H}$ NMR spectroscopy (Figure 4d). As the system was irradiated with blue light, the diagnostic signals from DHP were observed to decrease as the signals from encapsulated CPD increased. At each data point, the molar composition of the system was calculated by integrating the signal corresponding to $\mathrm{H}_{12}$ from DHP $(-6.44$ ppm) against $\mathrm{H}_{16}$ from CPD $(-0.60 \mathrm{ppm}$ ) (see also Figure 1c). This analysis allowed us to conclude that the reaction was near-quantitative, with up to $94 \%$ of CPDC1 at the photostationary state (PSS) (obtained within $<1 \mathrm{~h}$ for the 1 $\mathrm{mM}$ solution used for these NMR studies).

The resulting CPD 1 complex was characterized using $1 \mathrm{D}$ and 2D NMR spectroscopy. The chemical shifts of CPD protons $\mathrm{H}_{13}-\mathrm{H}_{15}$ were shifted upfield by $1.19-1.36 \mathrm{ppm}$, whereas the methyl group $\left(\mathrm{H}_{16}\right)$ experienced the largest shift, from $1.52 \mathrm{ppm}^{38}$ to $-0.60 \mathrm{ppm}$ (Figure S17). Similarly, the ${ }^{1} \mathrm{H}$ DOSY spectrum indicates that CPD and cage 1 diffuse at the same rate, which confirms the formation of an inclusion complex (Figure S18). Furthermore, ${ }^{1} \mathrm{H}-{ }^{1} \mathrm{H}$ COSY and ${ }^{1} \mathrm{H}-{ }^{1} \mathrm{H}$ NOESY spectra were used to assign all signals and confirm that $1: 1$ binding stoichiometry is maintained after isomerization (Figures S19 and S20). The NOESY correlations observed for CPD's methyl groups within CPDC1 were very similar to those for $D H P \subset 1$, which lead us to infer that they reside within the edges of the cage identified by the equatorial Pd atoms. In contrast to DHPC1, however, we found additional host-guest correlations, which involved all CPD protons (Figure S21).

Interestingly, $\mathrm{H}_{13}$ and $\mathrm{H}_{14}$ are correlated with $\mathrm{H}_{4}$ and $\mathrm{H}_{7}$ from the benzene and equatorial-imidazole regions of the cage. This observation suggests that the guest assumes a well-defined 

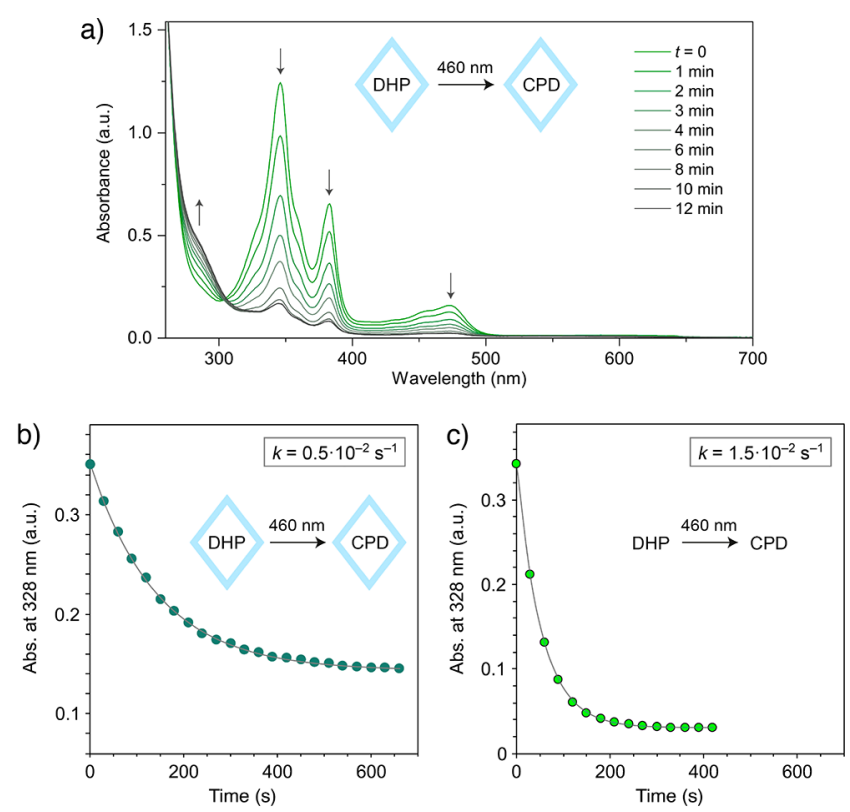

d)

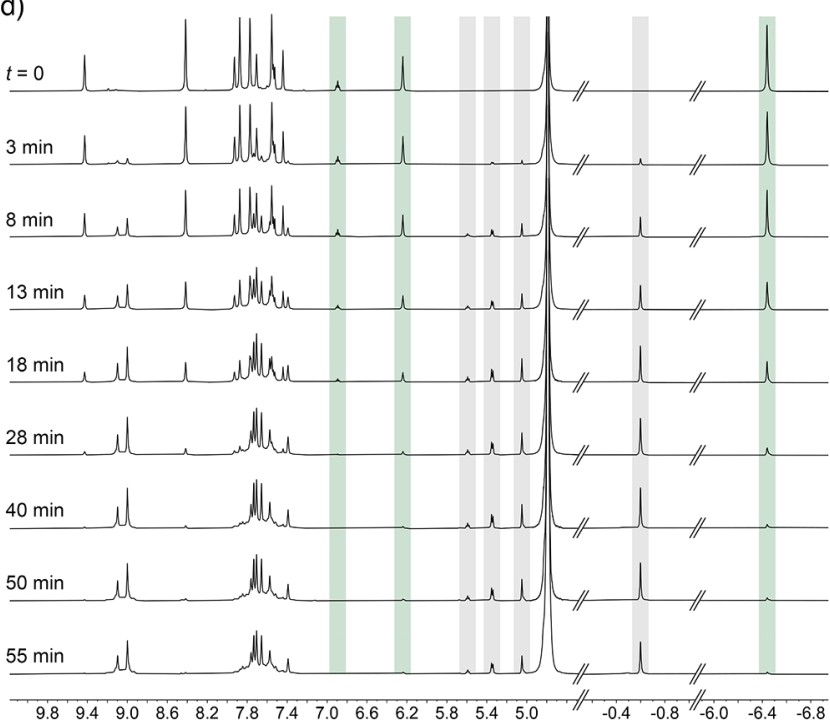

Figure 4. (a) A series of UV/vis absorption spectra of DHPC1 accompanying irradiation with $460 \mathrm{~nm}$ light. (b) Kinetics of photoisomerization of DHPC1 to CPDC1 in water at $20{ }^{\circ} \mathrm{C}$. (c) Kinetics of photoisomerization of DHP to CPD in acetonitrile at 20 ${ }^{\circ} \mathrm{C}$. Experiments in (b) and (c) were performed with the same irradiation setup $\left(\lambda_{\text {irr }}=460 \mathrm{~nm}\right)$ under a pseudo-first-order regime $\left(A_{460} \ll 1\right) .{ }^{47}$ Both time profiles were fitted with a first-order model (gray lines), and apparent first-order rate constants are reported. (d) Partial ${ }^{1} \mathrm{H}$ NMR spectra $\left(400 \mathrm{MHz}, \mathrm{D}_{2} \mathrm{O}\right)$ of DHPC1 recorded after different times of irradiation with $460 \mathrm{~nm}$ light. Signals highlighted in green and gray originate from DHP and CPD, respectively.

orientation, with protons $\mathrm{H}_{13}$ and $\mathrm{H}_{14}$ located within the open windows of the cage. Unfortunately, despite extensive efforts, we did not succeed in obtaining single crystals of CPD $\subset 1$. However, because of the remarkable agreement between the NOESY and X-ray data for DHPC1, we anticipate that the crystal structure of $C P D \subset 1$ would similarly reflect the solution-state structure determined by NMR.

To obtain further insight into the structures of DHPC1 and CPD $\subset 1$, we studied both inclusion complexes by atomistic molecular dynamics (MD) simulations in explicit water. ${ }^{39}$ Upon binding guests, cage 1 undergoes structural deformation, which can be quantified by two parameters: $d_{1}$, the distance between the axial Pd atoms, and $d_{2}$, the distance between the midpoints of opposite edges of the cage identified by the equatorial Pd atoms (see Figure 5a, inset). Figure 5a shows the
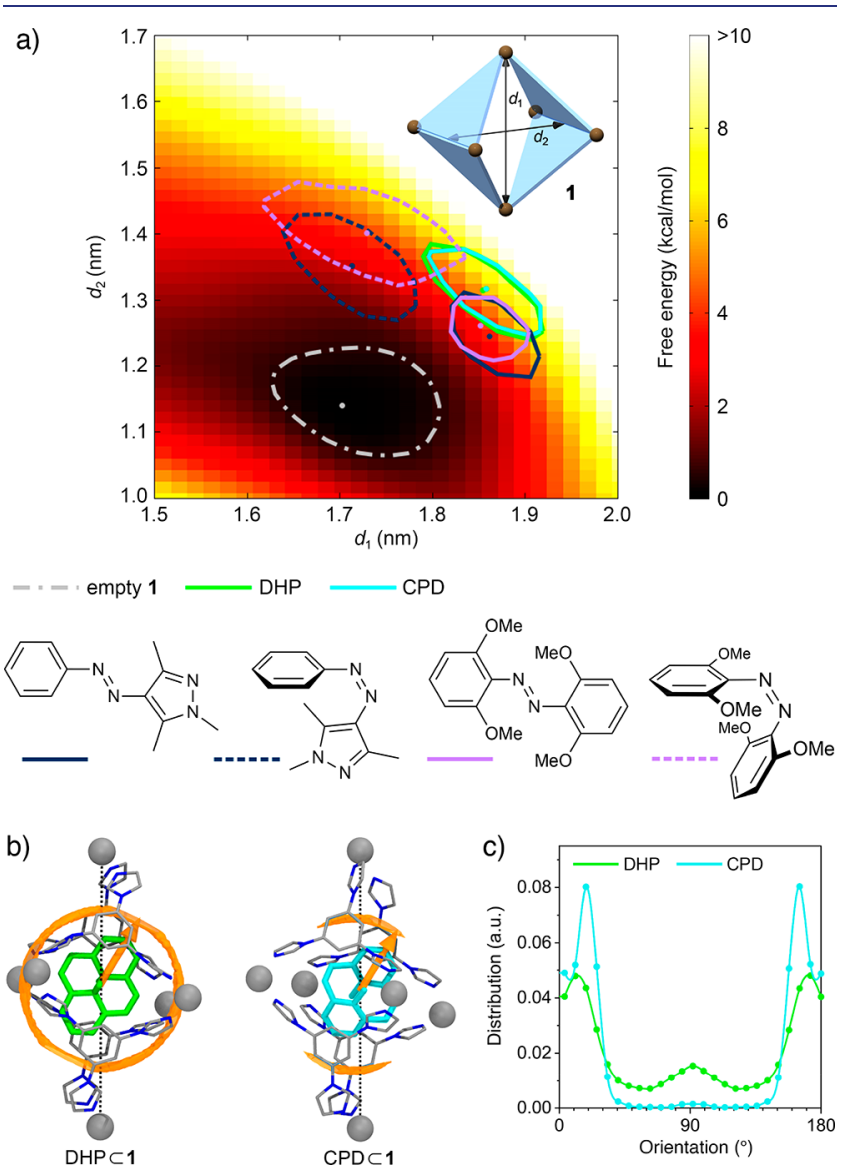

Figure 5. (a) Conformational free-energy landscape of empty cage 1, mapped as a function of the distance between the axial Pd atoms $\left(d_{1}\right)$ and the distance between the midpoints of opposite edges of the cage identified by the equatorial $\mathrm{Pd}$ atoms ( $d_{2}$; see inset) ${ }^{39}$ The position of the minimum-free-energy configuration of empty $\mathbf{1}$ is indicated with a gray point; the dash-dotted line identifies the region within $0.5 \mathrm{kcal} /$ mol of the global minimum. Projected on the free-energy surface of 1 are energy minima (colored points) and regions within $0.5 \mathrm{kcal} / \mathrm{mol}$ of the minima (lines) of DHPC1, CPDC1, and four other, representative inclusion complexes involving photochromic guests.

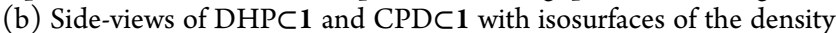
of guest's orientation (shown in orange). (c) Normalized distributions of guest orientations within DHPC1 and CPDC1.

conformational free-energy surface (FES) of empty $\mathbf{1},{ }^{39}$ where the gray dash-dotted line identifies the region within $0.5 \mathrm{kcal} /$ mol from the global-minimum free-energy configuration of the empty cage, denoted with a gray dot in the dark region of the FES. The green and cyan dots denote free-energy minima of the cage encapsulating DHP and CPD (i.e., DHPC1 and CPDC1), respectively. As we have recently demonstrated, such analysis of cage models provides information on the freeenergy cost associated with cage deformation upon guest encapsulation. ${ }^{39}$ In this case, our results suggest that this freeenergy cost amounts to $\sim 6 \mathrm{kcal} / \mathrm{mol}$ for both DHPC1 and CPDC1 (this cost is compensated by more favorable hostguest interactions, which lead to guest encapsulation). Remarkably, both the energy minima and the regions within 
$0.5 \mathrm{kcal} / \mathrm{mol}$ of the minima were found to be very similar for $\mathrm{DHP} \subset 1$ and CPDC1. This finding can be explained by the relatively minor conformational change associated with the isomerization of DHP to CPD. In sharp contrast, isomerization of other photochromic systems, such as arylazopyrazole (darkblue) and tetra-o-methoxyazobenzene (violet), was accompanied by large changes in both $d_{1}$ and $d_{2}$ (Figure 5a), ${ }^{39}$ in agreement with the large structural difference between the trans and cis isomers of these compounds.

Next, we used MD simulations to study the orientation and mobility of DHP and CPD guests inside cage 1 . The orange areas in Figure $5 b$ describe the trajectories of the tips of the orange arrows identifying the long axes of DHP and CPD (i.e., the lines connecting the two most distant carbon atoms of each guest). The larger is the orange area, the higher is the mobility of the guest within the cage during the equilibrium MD run. The orange ring in Figure $5 \mathrm{~b}$, left, means that the DHP guest can freely rotate inside the cage in a gyroscope-like fashion, ${ }^{40-43}$ with its two methyl groups interacting stably with the equatorial region of the cage. The facile rotation of DHP within 1 can be explained by the guest's structure, where the methyl groups protrude perpendicular with respect to the aromatic system. The interactions of these methyl groups with the equatorial region of the cage position the aromatic system of DHP precisely along the long axis of the cage (defined as the black dotted line connecting two axial Pd atoms in Figure $5 b)$, whereas the planarity of the aromatic system poses limited steric hindrance to rotation. In contrast, the CPD guest appears to be significantly more constrained in quasi-vertical configuration (in the $\mathrm{MD}$ regime), with the orange trajectories populated by its main axis located within $\sim 30^{\circ}$ of the long axis of the cage (Figure 5b, right). Analysis of a minimum-freeenergy configuration (Figure S24b) reveals that CPD's methyl groups interact with cage 1's edges and its benzene rings interact with the benzene rings of 1's imidazole panels. These interactions "lock" the CPD guest within the cage, which explains the NOESY correlations involving cage 1 with all protons from CPD (Figure S21).

The most favorable orientations of DHP and CPD within the cage were quantified by calculating the distributions of guest orientations, defined by the angle between the long axis of the guest (orange arrows in Figure $5 b$ ) and the long axis of the cage (black dotted line). The two maxima at $\sim 10^{\circ}$ and $\sim 170^{\circ}$ in the green curve in Figure $5 \mathrm{c}$ indicate that DHP preferentially assumes a quasi-vertical orientation within the cage. A smaller maximum at $\sim 90^{\circ}$ suggests that a horizontal orientation is also plausible. Remarkably, both of these guest configurations have also been identified in the crystal structure of DHPC1 (Figure 2c, left and right, respectively). On the other hand, the calculated orientation distributions for the $\mathrm{CPD}$ isomer (Figure 5c, blue line) confirm that it resides inside the cage exclusively in a near-vertical orientation.

Having extensively characterized the 1:1 inclusion complexes DHP $\subset 1$ and $\mathrm{CPD} \subset 1$, we investigated the reversibility of photoisomerization by ${ }^{1} \mathrm{H}$ NMR and UV/vis absorption spectroscopy (Figure 6). The back-isomerization reaction can be induced by irradiation with UV light at $254 \mathrm{~nm}$. Indeed, by exposing a solution of CPDC1 to $254 \mathrm{~nm}$, the signals corresponding to DHP were observed to re-emerge, and a new PSS comprising as much as $\sim 98 \%$ of $\mathrm{DHP} \subset \mathbf{1}$ was reached within $40 \mathrm{~min}$ (for the $1 \mathrm{mM}$ solution used in the NMR studies). Remarkably, we observed negligible cage decomposition, despite prolonged irradiation with highly energetic
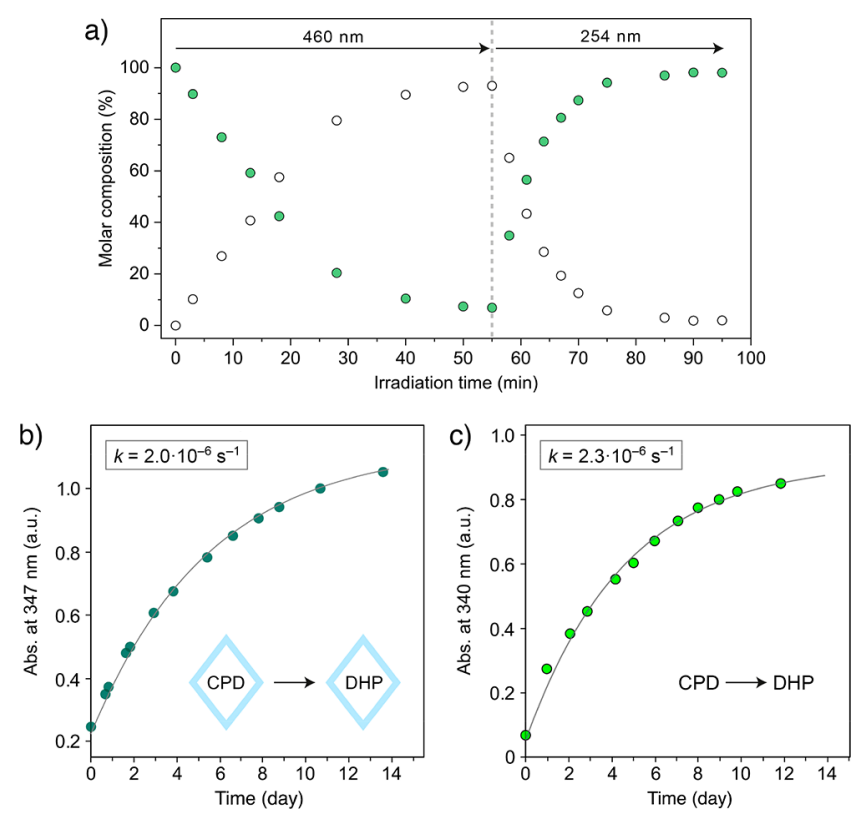

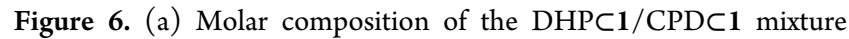
(green and empty markers, respectively) as a function of 460 and 254 $\mathrm{nm}$ irradiation time. The data are extracted from NMR spectra recorded immediately after irradiation (for the original spectra, see Figure S22). (b) Kinetics of the thermal back-isomerization of CPDC1 to DHPC1 in water at $20{ }^{\circ} \mathrm{C}$. (c) Kinetics of the thermal back-isomerization of CPD to DHP in acetonitrile at $20^{\circ} \mathrm{C}$. The data in both (b) and (c) are consistent with first-order kinetics, as indicated by the gray lines (data fitting).

UV light. These results indicate that the photoswitching is near-quantitative in both directions (Figure 6a). Although even higher conversions can be achieved for nonencapsulated DHP in acetonitrile, the reaction cycle is accompanied by a significant degree of decomposition (Figure S25).

The ring-closing reaction $(\mathrm{CPD} \rightarrow \mathrm{DHP})$ not only occurs upon irradiation with UV light but also spontaneously in the dark. To investigate the rate of this back-reaction, we followed

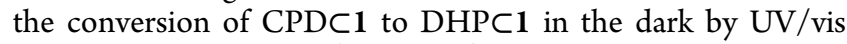
spectroscopy over time (Figure 6b); by comparing the spectral changes for solutions of CPDC1 in water and free CPD in acetonitrile (Figure $6 \mathrm{c}$ ), we found that the reaction in both cases obeyed first-order kinetics, with kinetic constants of $2.0 \times$ $10^{-6}$ and $2.3 \times 10^{-6} \mathrm{~s}^{-1}$, respectively. The negligible difference between these two values suggests that confinement within cage 1 does not significantly influence the rate of backisomerization. This finding is in agreement with our MD simulations, which suggest that the structural changes associated with isomerization from CPD to DHP can be readily accommodated within the cavity of cage 1 .

Because DHP isomerization is thought to proceed through a biradical species, ${ }^{6,7}$ the fatigue resistance of this process may be compromised by oxidative and radical-radical decomposition pathways. To determine if confinement within cage 1 would contribute additional stabilization to this reaction, we compared the fatigue experienced by encapsulated and free DHP over 10 isomerization cycles (Figure 7). Because DHP is insoluble in water, we chose to compare the behavior of aqueous DHPC1 with that of free DHP dissolved in pentane, which is stable under the conditions used for the cycling experiment and does not readily react with radical species. To 

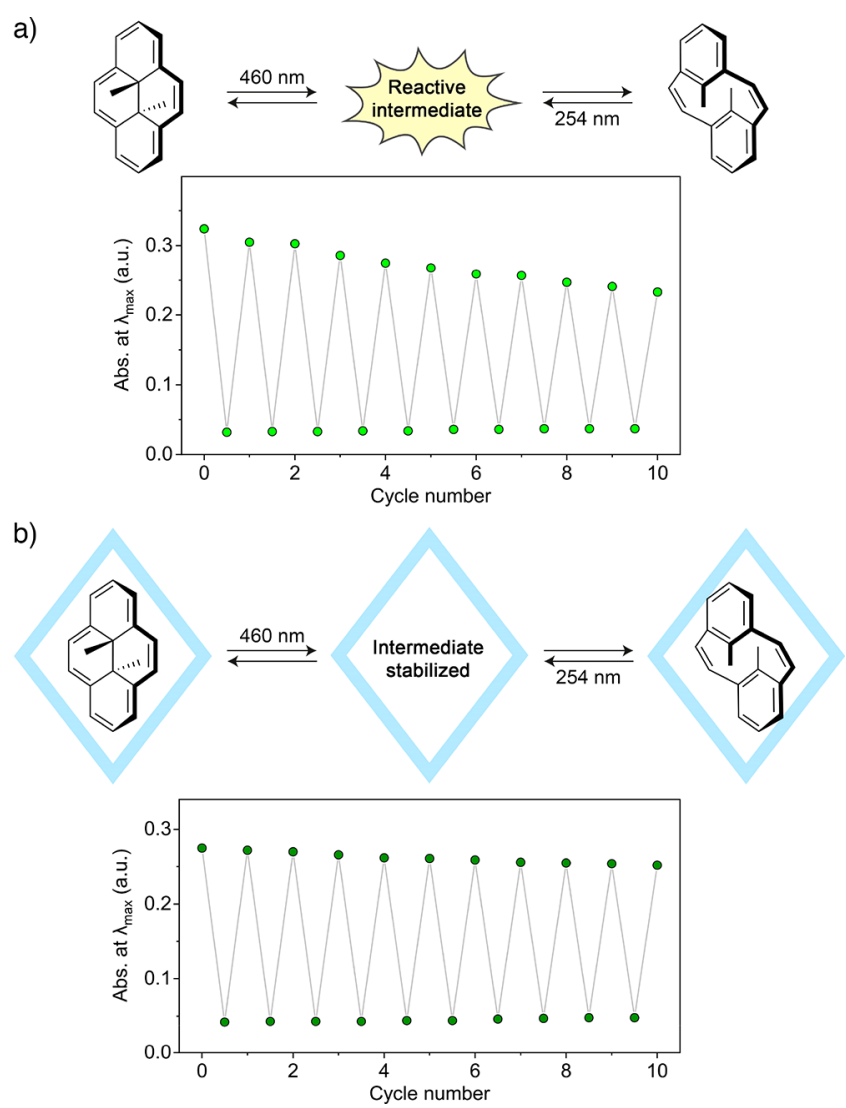

Figure 7. (a) Poor fatigue resistance of free DHP in pentane solution over 10 switching cycles. (b) Improved fatigue resistance of DHPC1 in water over 10 switching cycles. Deoxygenated solvents were used. In each cycle, the samples were exposed to $10 \mathrm{~min}$ of blue light $(\lambda=$ $460 \mathrm{~nm})$ followed by $10 \mathrm{~min}$ of UV light $(\lambda=254 \mathrm{~nm})$.

avoid the interference of oxygen in the switching process, the solvents were deoxygenated by bubbling $\mathrm{N}_{2}$.

Deoxygenated solutions of DHPC1 in water and DHP in pentane (at concentrations in the micromolar range) were prepared, and cyclic isomerization of the two solutions was achieved in parallel by irradiating both samples with blue light at $460 \mathrm{~nm}(10 \mathrm{~min})$, then UV light at $254 \mathrm{~nm}(10 \mathrm{~min})$; this process was followed by UV/vis spectroscopy. After 10 cycles, we calculated that $28 \%$ of free DHP in pentane was lost due to fatigue (Figure 7a). As anticipated, however, confinement within cage 1 contributed significantly to stabilizing the photochemical conversion between DHP and CPD. Only 8\% of encapsulated DHP was observed to decompose after completion of this experiment (Figure $7 \mathrm{~b}$ ). When the above experiment was repeated in water that had not previously been deoxygenated, $13 \%$ of DHP was observed to decompose after 10 irradiation cycles under otherwise identical conditions. The additional decomposition experienced by DHP in the presence of oxygen is unsurprising given the large open windows within the cage framework, which allow oxygen to easily access encapsulated photoswitch (Figure S26). Significantly, the fatigue experienced by DHP in this experiment remains far lower than the fatigue experienced by free DHP in deoxygenated pentane.

\section{CONCLUSIONS}

In this work, we have shown that DHP and CPD form 1:1 inclusion complexes with cage $\mathbf{1}$, and the flexibility of the cage allows the cavity to adapt in response to guest isomerization. Consequently, confinement within the cage does not significantly impact conversion between DHP and CPD, which could be realized with very high yields in both directions. Instead, confinement within cage $\mathbf{1}$ serves as a protective environment for the reactive intermediate involved in this reaction, minimizing bimolecular events and improving fatigue resistance during photoswitching cycles. In short, the cage protects DHP from photodegradation without compromising efficient photoswitching. The degree of stabilization is remarkable given the open structure of the cage and rapid exchange kinetics found for a structurally similar guest (sulfonated merocyanine). While confinement is known to contribute to stabilizing reactive species, previous examples typically utilize this effect for accelerating reactions or for trapping and visualizing transient species. ${ }^{44-46}$ Significantly, this work introduces the idea that stabilization within confined spaces can improve the yield of a reaction by preserving an unstable intermediate from degradation. While this particular system is limited to water, which drives encapsulation of DHP due to the hydrophobic effect (Supporting Information, section 14), this strategy can in principle be extended to confined environments in a wide variety of solvents. We envisage that confinement of DHP may improve its utility for applications requiring high-performance photoswitches. Furthermore, we anticipate that this approach is extensible to improving the yield of other reactions. In traditional organic chemistry, side-products are often generated due to undesirable reactions involving a reactive intermediate. When an inert environment is provided for such intermediates, confinement could thus provide a means to minimize side-products and improve reaction yield.

\section{ASSOCIATED CONTENT}

\section{Supporting Information}

The Supporting Information is available free of charge at https://pubs.acs.org/doi/10.1021/jacs.0c06146.

General information, synthesis and characterization of triimidazole ligand, synthesis and characterization of cage 1, synthesis and characterization of DHP, formation and characterization of $\mathrm{DHP} \subset 1, \mathrm{UV} /$ vis titration of cage 1 with DHP, X-ray data collection and structure refinement of $\mathrm{DHPC1}$, comparison of the crystal structures of $\mathrm{DHP} \subset 1$ and $\mathrm{MCC}$, guest exchange experiments, formation and characterization of CPDC1, molecular dynamics simulations, photoisomerization study of free DHP in acetonitrile, photoisomerization study of $\mathrm{DHP} \subset 1$ in nondeoxygenated water, study of release of DHP from cage 1 using acetonitrile, and supplementary references (PDF)

X-ray crystal structure of DHPC1 (CIF)

\section{AUTHOR INFORMATION}

\section{Corresponding Author}

Rafal Klajn - Department of Organic Chemistry, Weizmann Institute of Science, Rehovot 76100, Israel; 이이이.org/00000002-6320-8875; Email: rafal.klajn@weizmann.ac.il

\section{Authors}

Martina Canton - Department of Organic Chemistry, Weizmann Institute of Science, Rehovot 76100, Israel; Center for Light-Activated Nanostructures (CLAN) and Dipartimento 
di Chimica Industriale, Università di Bologna, Bologna 40136, Italy

Angela B. Grommet - Department of Organic Chemistry, Weizmann Institute of Science, Rehovot 76100, Israel

Luca Pesce - Department of Innovative Technologies, University of Applied Sciences and Arts of Southern Switzerland, Manno CH-6928, Switzerland; 이이.org/0000-0001-6364-9577

Julius Gemen - Department of Organic Chemistry, Weizmann Institute of Science, Rehovot 76100, Israel

Shiming Li - Department of Chemistry and Chemical Engineering, Chalmers University of Technology, Göteborg 41296, Sweden

Yael Diskin-Posner - Chemical Research Support, Weizmann Institute of Science, Rehovot 76100, Israel; (1) orcid.org/00000002-9008-8477

Alberto Credi - Center for Light-Activated Nanostructures (CLAN) and Dipartimento di Chimica Industriale, Università di Bologna, Bologna 40136, Italy; 이 orcid.org/0000-00032546-9801

Giovanni M. Pavan - Department of Innovative Technologies, University of Applied Sciences and Arts of Southern Switzerland, Manno CH-6928, Switzerland; Department of Applied Science and Technology, Politecnico di Torino, Torino 10129, Italy; (1) orcid.org/0000-0002-3473-8471

Joakim Andréasson - Department of Chemistry and Chemical Engineering, Chalmers University of Technology, Göteborg 41296, Sweden; (1) orcid.org/0000-0003-4695-7943

Complete contact information is available at:

https://pubs.acs.org/10.1021/jacs.0c06146

\section{Author Contributions}

${ }^{\nabla}$ M.C. and A.B.G. contributed equally.

\section{Notes}

The authors declare no competing financial interest.

\section{ACKNOWLEDGMENTS}

We acknowledge funding from the European Union's Horizon 2020 research and innovation program under the European Research Council (ERC) grant agreements 820008 (to R.K.), 818776 (to G.M.P.), and 692981 (to A.C.) and the Marie Skłodowska-Curie grant agreement no. 812868 (to J.G.), the Swedish Research Council VR (grant agreement 2016-03601 to J.A.), the Swiss National Science Foundation (grant agreement 200021175735 to G.M.P.), and the Minerva Foundation with funding from the Federal German Ministry for Education and Research (R.K.). L.P. and G.M.P. acknowledge the computational resources provided by the Swiss National Supercomputing Center (CSCS). A.B.G. acknowledges funding from the Zuckerman STEM Leadership Program.

\section{REFERENCES}

(1) Bohne, C.; Mitchell, R. H. Characterization of the Photochromism of Dihydropyrenes with Photophysical Techniques. J. Photochem. Photobiol., C 2011, 12, 126-137.

(2) Boelke, J.; Hecht, S. Designing Molecular Photoswitches for Soft Materials Applications. Adv. Opt. Mater. 2019, 7, 1900404.

(3) Garmshausen, Y.; Klaue, K.; Hecht, S. Dihydropyrene as an Aromaticity Probe for Partially Quinoid Push-Pull Systems. ChemPlusChem 2017, 82, 1025-1029.

(4) Ayub, K.; Zhang, R.; Robinson, S. G.; Twamley, B.; Williams, R. V.; Mitchell, R. H. Suppressing the Thermal Metacyclophanediene to Dihydropyrene Isomerization: Synthesis and Rearrangement of 8,16-
Dicyano[2.2]Metacyclophane-1,9-Diene and Evidence Supporting the Proposed Biradicaloid Mechanism. J. Org. Chem. 2008, 73, 451-456. (5) Ayub, K.; Li, R.; Bohne, C.; Williams, R. V.; Mitchell, R. H. Calculation Driven Synthesis of an Excellent Dihydropyrene Negative Photochrome and Its Photochemical Properties. J. Am. Chem. Soc. 2011, 133, 4040-4045.

(6) Sheepwash, M. A. L.; Mitchell, R. H.; Bohne, C. Mechanistic Insights into the Photochromism of trans-10b,10c-Dimethyl-10b,10cdihydropyrene Derivatives. J. Am. Chem. Soc. 2002, 124, 4693-4700.

(7) Liddell, P. A.; Kodis, G.; Andréasson, J.; De La Garza, L.; Bandyopadhyay, S.; Mitchell, R. H.; Moore, T. A.; Moore, A. L.; Gust, D. Photonic Switching of Photoinduced Electron Transfer in a Dihydropyrene-Porphyrin-Fullerene Molecular Triad. J. Am. Chem. Soc. 2004, 126, 4803-4811.

(8) Grommet, A. B.; Feller, M.; Klajn, R. Chemical Reactivity under Nanoconfinement. Nat. Nanotechnol. 2020, 15, 256-271.

(9) Yoshizawa, M.; Klosterman, J. K.; Fujita, M. Functional Molecular Flasks: New Properties and Reactions within Discrete, Self-Assembled Hosts. Angew. Chem., Int. Ed. 2009, 48, 3418-3438.

(10) Cook, T. R.; Stang, P. J. Recent Developments in the Preparation and Chemistry of Metallacycles and Metallacages via Coordination. Chem. Rev. 2015, 115, 7001-7045.

(11) Pullen, S.; Clever, G. H. Mixed-Ligand Metal-Organic Frameworks and Heteroleptic Coordination Cages as Multifunctional Scaffolds-A Comparison. Acc. Chem. Res. 2018, 51, 3052-3064.

(12) Jongkind, L. J.; Caumes, X.; Hartendorp, A. P. T.; Reek, J. N. H. Ligand Template Strategies for Catalyst Encapsulation. Acc. Chem. Res. 2018, 51, 2115-2128.

(13) Sepehrpour, H.; Fu, W.; Sun, Y.; Stang, P. J. Biomedically Relevant Self-Assembled Metallacycles and Metallacages. J. Am. Chem. Soc. 2019, 141, 14005-14020.

(14) Ward, M. D.; Hunter, C. A.; Williams, N. H. Coordination Cages Based on Bis(Pyrazolylpyridine) Ligands: Structures, Dynamic Behavior, Guest Binding, and Catalysis. Acc. Chem. Res. 2018, 51, 2073-2082.

(15) Li, H.; Zhu, Z.; Fahrenbach, A. C.; Savoie, B. M.; Ke, C.; Barnes, J. C.; Lei, J.; Zhao, Y.-L.; Lilley, L. M.; Marks, T. J.; Ratner, M. A.; Stoddart, J. F. Mechanical Bond-Induced Radical Stabilization. J. Am. Chem. Soc. 2013, 135, 456-467.

(16) Yamada, M.; Kurihara, H.; Suzuki, M.; Saito, M.; Slanina, Z.; Uhlik, F.; Aizawa, T.; Kato, T.; Olmstead, M. M.; Balch, A. L.; Maeda, Y.; Nagase, S.; Lu, X.; Akasaka, T. Hiding and Recovering Electrons in a Dimetallic Endohedral Fullerene: Air-Stable Products from Radical Additions. J. Am. Chem. Soc. 2015, 137, 232-238.

(17) Geer, M. F.; Walla, M. D.; Solntsev, K. M.; Strassert, C. A.; Shimizu, L. S. Self-Assembled Benzophenone Bis-Urea Macrocycles Facilitate Selective Oxidations by Singlet Oxygen. J. Org. Chem. 2013, 78, 5568-5578.

(18) Coles, M. S.; Quach, G.; Beves, J. E.; Moore, E. G. A Photophysical Study of Sensitization-Initiated Electron Transfer: Insights into the Mechanism of Photoredox Activity. Angew. Chem., Int. Ed. 2020, 59, 9522-9526.

(19) Howlader, P.; Mondal, B.; Purba, P. C.; Zangrando, E.; Mukherjee, P. S. Self-Assembled Pd(II) Barrels as Containers for Transient Merocyanine Form and Reverse Thermochromism of Spiropyran. J. Am. Chem. Soc. 2018, 140, 7952-7960.

(20) Takezawa, H.; Akiba, S.; Murase, T.; Fujita, M. Cavity-Directed Chromism of Phthalein Dyes. J. Am. Chem. Soc. 2015, 137, 70437046.

(21) Dolgopolova, E. A.; Berseneva, A. A.; Faillace, M. S.; Ejegbavwo, O. A.; Leith, G. A.; Choi, S. W.; Gregory, H. N.; Rice, A. M.; Smith, M. D.; Chruszcz, M.; Garashchuk, S.; Mythreye, K.; Shustova, N. B. Confinement-Driven Photophysics in Cages, Covalent-Organic Frameworks, Metal-Organic Frameworks, and DNA. J. Am. Chem. Soc. 2020, 142, 4769-4783.

(22) Baggi, G.; Casimiro, L.; Baroncini, B.; Silvi, S.; Credi, A.; Loeb, $\mathrm{S}$. J. Threading-gated photochromism in [2]pseudorotaxanes. Chem. Sci. 2009, 10, 5104-5113. 
(23) Deniz, E.; Tomasulo, M.; Cusido, J.; Sortino, S.; Raymo, F. Fast and Stable Photochromic Oxazines for Fluorescence Switching. Langmuir 2011, 27, 11773-11783.

(24) Beaujean, P.; Bondu, F.; Plaquet, A.; Garcia-Amorós, J.; Cusido, J.; Raymo, F. M.; Castet, F.; Rodriguez, V.; Champagne, B. Oxazines: A New Class of Second-Order Nonlinear Optical Switches. J. Am. Chem. Soc. 2016, 138, 5052-5056.

(25) Mallo, N.; Brown, P. T.; Iranmanesh, H.; MacDonald, T. S. C.; Teusner, M. J.; Harper, J. B.; Balla, G. E.; Beves, J. Photochromic switching behaviour of donor-acceptor Stenhouse adducts in organic solvents. Chem. Commun. 2016, 52, 13576-13579.

(26) Juríček, M.; Strutt, N. L.; Barnes, J. C.; Butterfield, A. M.; Dale, E. J.; Baldridge, K. K.; Stoddart, J. F.; Siegel, J. S. Induced-Fit Catalysis of Corannulene Bowl-to-Bowl Inversion. Nat. Chem. 2014, 6, 222228.

(27) Mal, P.; Breiner, B.; Rissanen, K.; Nitschke, J. R. White Phosphorus Is Air-Stable Within a Self-Assembled Tetrahedral Capsule. Science 2009, 324, 1697-1699.

(28) Rizzuto, F. J.; Ramsay, W. J.; Nitschke, J. R. Otherwise Unstable Structures Self-Assemble in the Cavities of Cuboctahedral Coordination Cages. J. Am. Chem. Soc. 2018, 140, 11502-11509.

(29) Sawada, T.; Yoshizawa, M.; Sato, S.; Fujita, M. Minimal Nucleotide Duplex Formation in Water through Enclathration in SelfAssembled Hosts. Nat. Chem. 2009, 1, 53-56.

(30) Yamashina, M.; Sei, Y.; Akita, M.; Yoshizawa, M. Safe Storage of Radical Initiators within a Polyaromatic Nanocapsule. Nat. Commun. 2014, 5, 4662.

(31) Cram, D. J.; Tanner, M. E.; Thomas, R. The Taming of Cyclobutadiene. Angew. Chem., Int. Ed. Engl. 1991, 30, 1024-1027.

(32) Warmuth, R. o-Benzyne: Strained Alkyne or Cumulene?NMR Characterization in a Molecular Container. Angew. Chem., Int. Ed. Engl. 1997, 36, 1347-1350.

(33) Samanta, D.; Galaktionova, D.; Gemen, J.; Shimon, L. J. W.; Diskin-Posner, Y.; Avram, L.; Král, P.; Klajn, R. Reversible Chromism of Spiropyran in the Cavity of a Flexible Coordination Cage. Nat. Commun. 2018, 9, 641.

(34) Samanta, D.; Gemen, J.; Chu, Z.; Diskin-Posner, Y.; Shimon, L. J. W.; Klajn, R. Reversible Photoswitching of Encapsulated Azobenzenes in Water. Proc. Natl. Acad. Sci. U. S. A. 2018, 115, 9379-9384.

(35) Hanopolskyi, A. I.; De, S.; Białek, M. J.; Diskin-Posner, Y.; Avram, L.; Feller, M.; Klajn, R. Reversible Switching of Arylazopyrazole within a Metal-Organic Cage. Beilstein J. Org. Chem. 2019, 15, 2398-2407.

(36) Samanta, D.; Mukherjee, S.; Patil, Y. P.; Mukherjee, P. S. SelfAssembled $\mathrm{Pd}_{6}$ Open Cage with Triimidazole Walls and the Use of Its Confined Nanospace for Catalytic Knoevenagel- and Diels-Alder Reactions in Aqueous Medium. Chem. - Eur. J. 2012, 18, 1232212329.

(37) Hou, L.; Leydecker, T.; Zhang, X.; Rekab, W.; Herder, M.; Cendra, C.; Hecht, S.; McCulloch, I.; Salleo, A.; Orgiu, E.; Samorì, P. Engineering Optically Switchable Transistors with Improved Performance by Controlling Interactions of Diarylethenes in Polymer Matrices. J. Am. Chem. Soc. 2020, 142, 11050-11059.

(38) Blattmann, H.-R.; Meuche, D.; Heilbronner, E.; Molyneux, R. J.; Boekelheide, V. Photoisomerization of trans-15,16-Dimethyldihydropyrene. J. Am. Chem. Soc. 1965, 87, 130-131.

(39) Pesce, L.; Perego, C.; Grommet, A. B.; Klajn, R.; Pavan, G. M. Molecular Factors Controlling the Isomerization of Azobenzenes in the Cavity of a Flexible Coordination Cage. J. Am. Chem. Soc. 2020, 142, 9792-9802.

(40) Commins, P.; Garcia-Garibay, M. A. Photochromic Molecular Gyroscope with Solid State Rotational States Determined by an Azobenzene Bridge. J. Org. Chem. 2014, 79, 1611-1619.

(41) Khuong, T.-A. V; Nuñez, J. E.; Godinez, C. E.; Garcia-Garibay, M. A. Crystalline Molecular Machines: A Quest Toward Solid-State Dynamics and Function. Acc. Chem. Res. 2006, 39, 413-422.

(42) Nawara, A. J.; Shima, T.; Hampel, F.; Gladysz, J. A. Gyroscopelike Molecules Consisting of $\mathrm{PdX}_{2} / \mathrm{PtX}_{2}$ Rotators Encased in Three-
Spoke Stators: Synthesis via Alkene Metathesis, and Facile Substitution and Demetalation. J. Am. Chem. Soc. 2006, 128, 49624963.

(43) Lang, G. M.; Shima, T.; Wang, L.; Cluff, K. J.; Skopek, K.; Hampel, F.; Blümel, J.; Gladysz, J. A. Gyroscope-Like Complexes Based on Dibridgehead Diphosphine Cages That Are Accessed by Three-Fold Intramolecular Ring Closing Metatheses and Encase $\mathrm{Fe}(\mathrm{CO})_{3}, \mathrm{Fe}(\mathrm{CO})_{2}(\mathrm{NO})^{+}$, and $\mathrm{Fe}(\mathrm{CO})_{3}(\mathrm{H})^{+}$Rotators. J. Am. Chem. Soc. 2016, 138, 7649-7663.

(44) Yoshizawa, M.; Kusukawa, T.; Fujita, M.; Yamaguchi, K. Shipin-a-Bottle Synthesis of Otherwise Labile Cyclic Trimers of Siloxanes in a Self-Assembled Coordination Cage. J. Am. Chem. Soc. 2000, 122, 6311-6312.

(45) Lee, T.-C.; Kalenius, E.; Lazar, A. I.; Assaf, K. I.; Kuhnert, N.; Grün, C. H.; Jänis, J.; Scherman, O. A.; Nau, W. M. Chemistry inside Molecular Containers in the Gas Phase. Nat. Chem. 2013, 5, 376382.

(46) Iwasawa, T.; Hooley, R. J.; Rebek, J. Stabilization of Labile Carbonyl Addition Intermediates by a Synthetic Receptor. Science 2007, 317, 493-496.

(47) Logan, S. R. Does a Photochemical Reaction Have a Reaction Order? J. Chem. Educ. 1997, 74, 1303. 